\title{
Présence de
}

\section{Bombus argillaceus SCOPOLI I763 (Apoidae - Apidae - Bombini) dans les Hautes-Alpes en France}

\author{
par Bernard FRIN*
}

Résumé. La difficulté qui existe dans la nature pour déterminer les espèces de Bombus parmi les espèces françaises m'a amené à choisir les reines de Bombus argillaceus, espèce polylectique, remarquable et facile à identifier même sur photo. Cette espèce atteint sa limite occidentale de distribution géographique dans nos vallées alpines où sa présence et sa répartition sont mal connues. Les résultats de cette enquête menée essentiellement sur les saisons 2013 et 2014 montrent que Bombus argillaceus (Scopoli 1763) est bien présent dans toutes les vallées des Hautes-Alpes, de l'étage collinéen à l'étage montagnard jusqu'à $1700 \mathrm{~m}$ environ. L'espèce est aussi attestée dans la vallée de l'Ubaye dans les Alpes-de-Haute-Provence. Seuls les secteurs du Serrois, du Devoluy, du Briançonnais et du Queyras sont restés vierges d'observations à ce

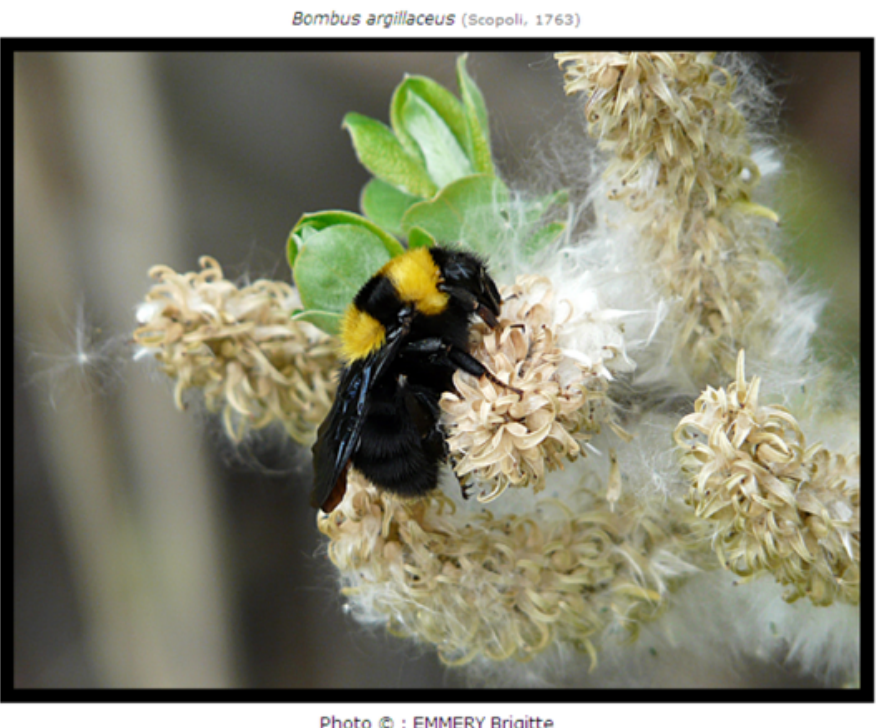

Figure I. Bombus argillaceus sur Salix sp. (Salicaceae). jour. Ceci est sans doute dû à une sousprospection de ces parties des Alpes françaises.

\begin{abstract}
As Bombus species are difficult to identify to the species level to the naked eyes, I chose to focus my observations on the queens of Bombus argillaceus among the French species. This is a remarkable species able to feed on various plants species (polylectic) and easily recognisable even on pictures. This species reaches its western limit in alpine valleys of France where its presence and distribution are not very well known. The results of our field prospections, mostly conducted during the 2013 and 2014 seasons, show that this species is well established in all the High-Alps valleys, as well as in the Ubaye Valley (Alpes-de-Haute-Provence) - up to about 1700 meters. To this day, we don't have any data in the areas of Serre, Devoluy, Queyras and Briançon, probably due to the low sampling effort in these regions.
\end{abstract}

\section{Introduction}

Je fus fort intrigué lorsque pour la première fois (le 9 avril 2009 à Châteauroux-les-Alpes) j’ai rencontré ce magnifique bourdon. Mon esprit s'est mis à vagabonder, me faisant tout d'abord penser avec ses ailes noires à une espèce de

*29 rue des Vignes, 05200 Embrun - be.frin@wanadoo.fr
Xylocopa, puis à un bourdon " coucou ». Rentré chez moi, je pus, après quelques recherches, enfin mettre un nom sur le spécimen capturé : Bombus argillaceus (figures 2 et 3). Membre du GRENHA (GRoupement des ENtomologistes des Hautes-Alpes) depuis sa création, je propose en septembre 2009 de commencer une collection du genre Bombus dans le département afin d'en faire l'inventaire. Depuis cette période, j'ai 


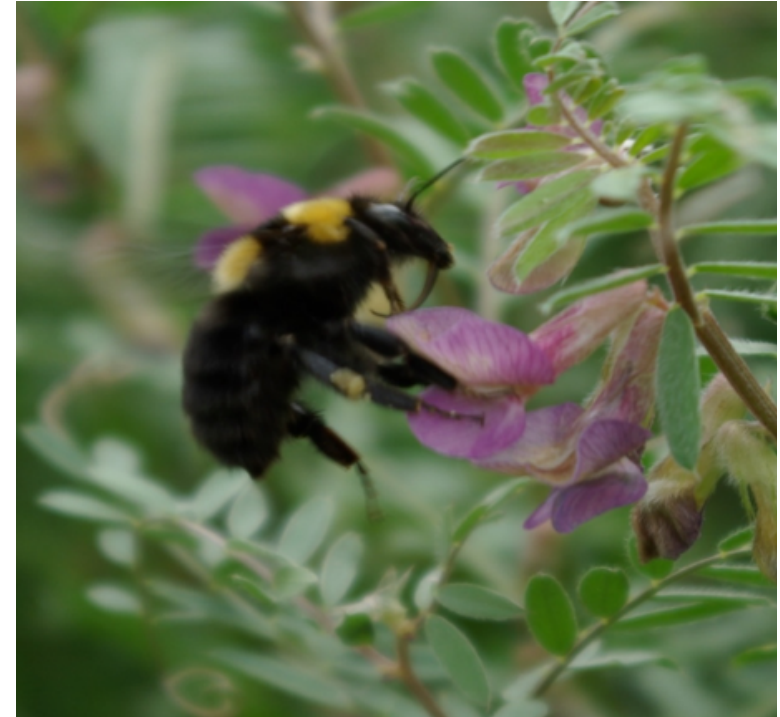

Figure 2. Bombus argillaceus sur Vicia pannonica (Fabaceae). (Photo : B. Frin)

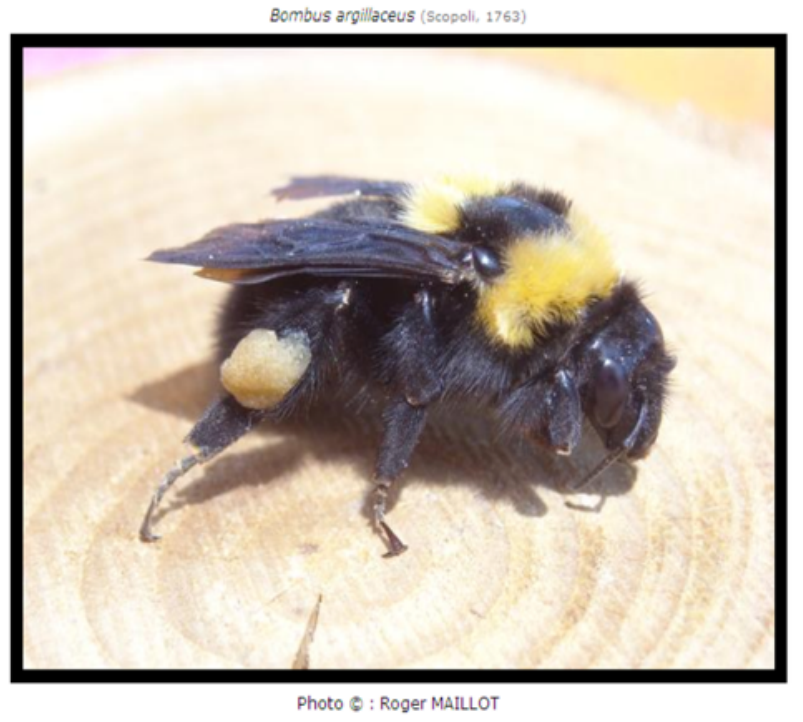

Figure 3. Reine Bombus argillaceus avec pelotes.

réalisé plein de belles découvertes dans ce genre mais également dans beaucoup d'autres genres d'Apoidea et ma collection compte actuellement 37 espèces de Bombus. C'est dans ce contexte que je suis aussi devenu membre et acteur de l'Observatoire des Abeilles (OA). Dans ce récit naturaliste, je vous présente certaines de mes observations autour de l'aire de répartition de Bombus argillaceus dans les vallées alpines françaises.

\section{Matériel et méthodes}

Début 2013, je décide de lancer une enquête sur Bombus argillaceus dans le département des Hautes-Alpes. A cet effet, je sollicite toutes mes relations naturalistes pour noter avec précision toutes leurs observations de reine de cette espèce et prendre des clichés. Les outils utilisés sont la clé des bourdons du massif armoricain par Gilles MAHÉ (2011) et la clé de détermination du Pr. Pierre RASMONT. J'ai également beaucoup utilisé le site Atlas Hymenoptera (RASMONT P. et ISERBYT S. 2010-2013). Des spécimens de références sont présents dans ma collection personnelle dans laquelle chacune des identifications ont été contrôlées par G. Mahé. L'ensemble des données sont disponibles puisque la saisie de toutes les observations a été faite dans la base de données du GRENHA, de laquelle sont extraits les documents qui sont inclus dans cette enquête.

\section{Pattern}

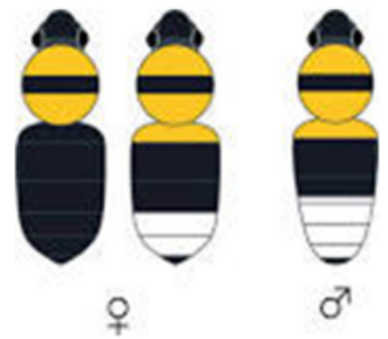

Figure 4. Pattern chez Bombus argillaceus.

\section{Résultats}

La consultation des deux cartes (figures 5 et 6 ) ci-dessus et la répartition altitudinale (figure 7)

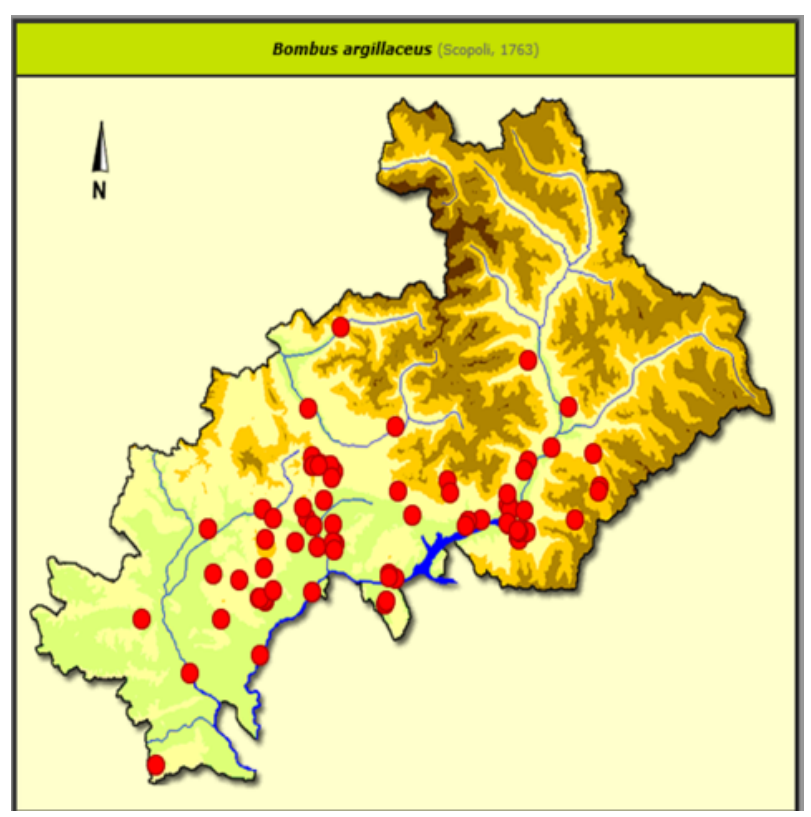

Figure 5. Observations dans les bassins fluviaux. 


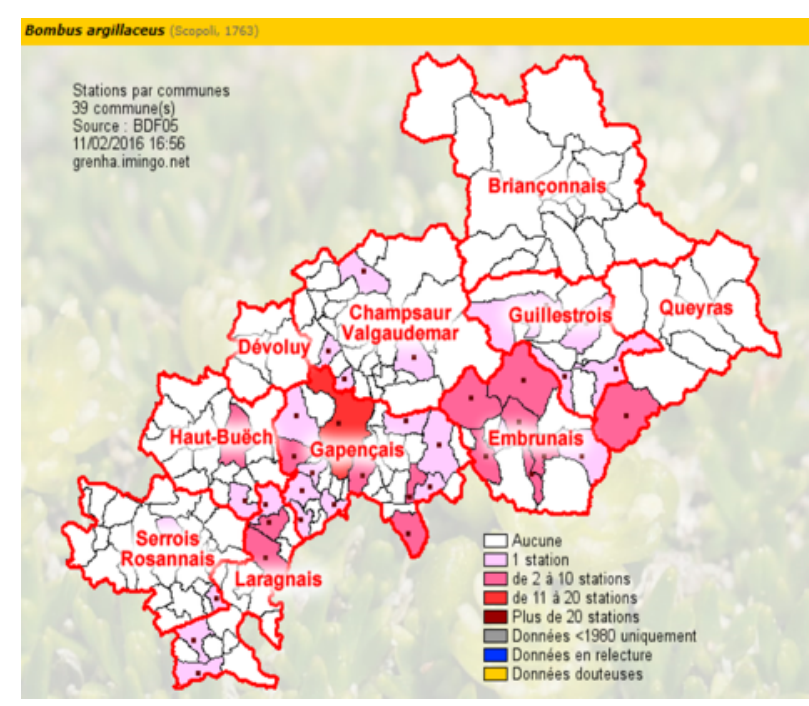

Figure 6. Communes et secteurs des Haute-Alpes.

permettent d'affirmer que $B$. argillaceus est bien présent dans toutes les vallées des HautesAlpes : Durance, Buëch, Drac (à un degré moindre) et celle de l'Ubaye dans le département voisin, les Alpes- de-Haute-Provence.

Les 92 observations enregistrées dans la carte communale (figure 6) couvrent 39 communes sur les 177 qui constituent le département. Les communes restées vierges d'observations et qui sont entourées par d'autres communes ou l'espèce a été observée, sont très certainement occupées bien que cela reste à vérifier car ces absences sont sûrement dû à un manque de pression d'observation. L'espèce occupe tout l'étage collinéen ainsi que l'étage montagnard dans sa partie, peu ou non boisée (l'observation la plus haute se situe à $1710 \mathrm{~m}$ ).

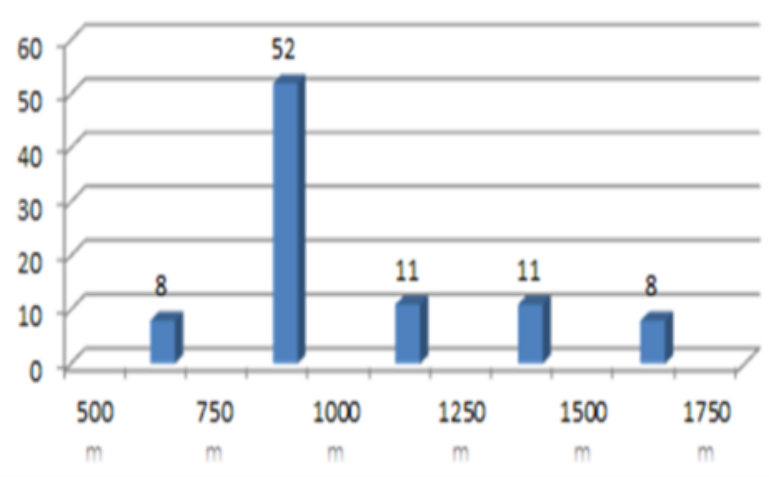

Figure 7. Répartition altitudinale des observations. Point le plus bas du département : 470 m Ribiers.

Enfin, nous pouvons noter l'absence d'observations de $B$. argillaceus dans les zones amont de la vallée de la Durance au nord, le
Briançonnais ainsi que dans le Queyras (haute vallée du Guil) puis à l'ouest dans le Serrois et le Dévoluy. Cela n'exclut pas la présence de l'espèce sur ces secteurs qui ont été très largement sous prospectés.

\section{Phénologie}

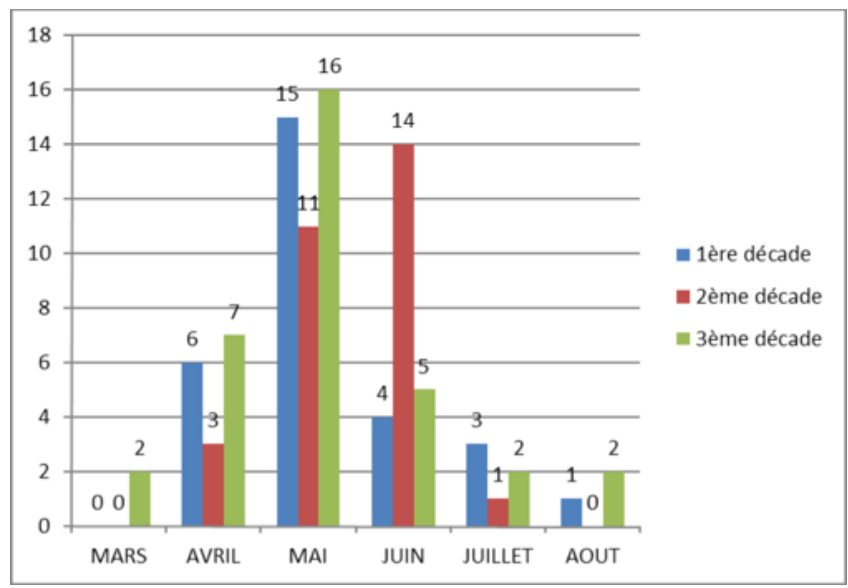

Figure 8. Phénologie de Bombus argillaceus.

L'émergence de l'espèce débute vers la fin mars (l'observation la plus précoce le 26.III.2011). Les observations continuent jusqu'à la deuxième décade de juin avec la plus grande partie (42 d'entre elles) au cours du mois de mai. L'observation la plus septentrionale a été réalisée sur la commune de Freissinières, au lieu-dit Les Aujards (4445’56.2” N, 6³1’34.3”E).

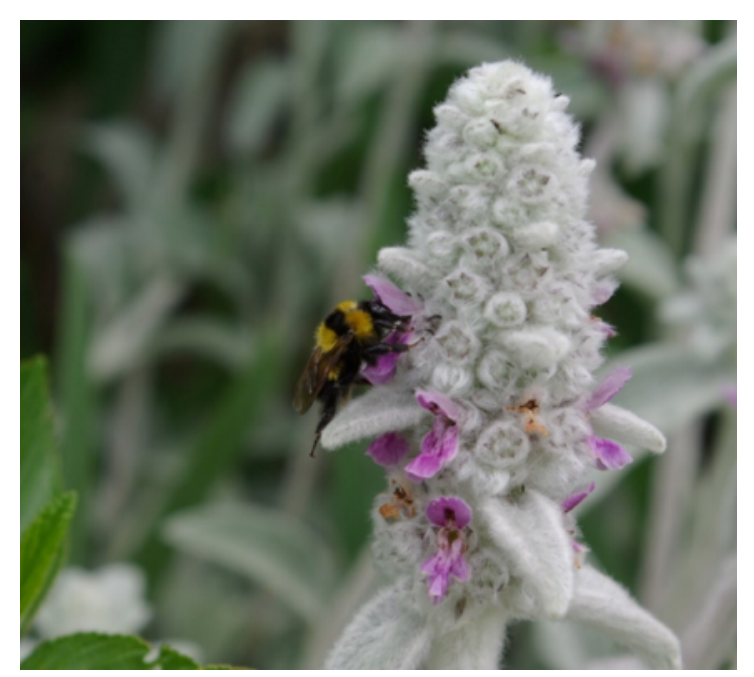

Figure 9. Mâle de B. argillaceus sur Stachys sp. (photo:B. Frin)

\section{Remarques}

Une seule ouvrière a été observée au cours de la première décade d'août (obs. pers. David 
Genoud). Deux données : une à Espinasses (18.VII.2012, Bernard Dardenne) et une à Embrun (24.VIII.2010 B. Frin), concernent des mâles collectés et identifiés car ils peuvent être confondus avec $B$. hortorum (figure 9). Une jeune reine a été observée pendant la troisième décade d'août 2014.

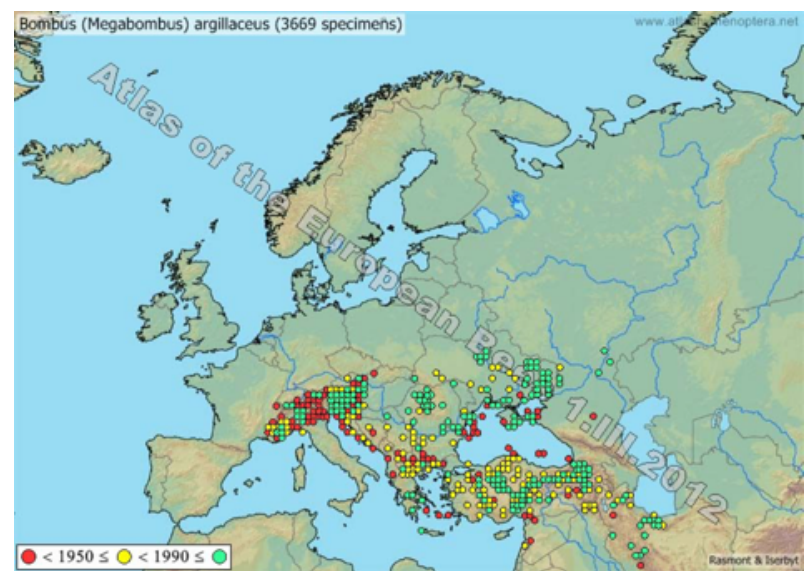

Figure 10. Bombus argillaceus en Europe et Asie.

\section{Discussion}

Le département des Hautes-Alpes est très riche par sa biodiversité et les abeilles ne font pas exception avec environ 300 espèces recensées fin 2016 (données non publiées). Parmi les espèces de Bombus qui fréquentent le département, on peut noter : $B$. gerstaeckeri, B. mendax, $B$. brodmannicus, $B$. sicheli, entre autres. $B$. argillaceus atteint dans les vallées alpines sa limite ouest de répartition (figure 10) et sa distribution mériterait d'être surveillée dans le cadre des changements climatiques en cours. De plus, l'espèce reste à rechercher dans le Briançonnais, le Queyras et le Dévoluy. Enfin nous pouvons nous poser la question du faible nombre d'ouvrière détectées ce qui peut venir du fait de la difficulté à les reconnaître avec certitude. De plus, la petite taille des colonies est aussi une des causes probables de cette faible détection. Enfin la dernière explication vient de notre protocole puisque celui-ci était ciblé sur les reines. Dans le futur nous pourront focaliser nos recherches sur les ouvrières principalement pendant la période estivale en juillet et août.

\section{Remerciements}

Mes remerciements s'adressent à tous les observateurs (Allombert Sylvain, Bernard Pierre, Boutin Michel, Braud Yoan, Brugot Didier, Classen Raphaël, Coulon Mireille, Dardenne
Bernard, Drouet Eric, Dusoulier François, Emmery Brigitte, Filosa Anne, Gagnaire Gilles, Genoud David, Maillot Roger, Paquet Isabelle, Raillot Jean, Rolland Blandine) et plus particulièrement à $\mathrm{F}$. Dusoulier (conservateur du Muséum d'histoire naturelle de Toulon et du Var) qui a bien voulu relire et corriger cette enquête et à Gilles Mahé pour le contrôle des déterminations des espèces de Bombus ainsi qu'aux membres de l'OA qui m'ont encouragé pour cette étude.

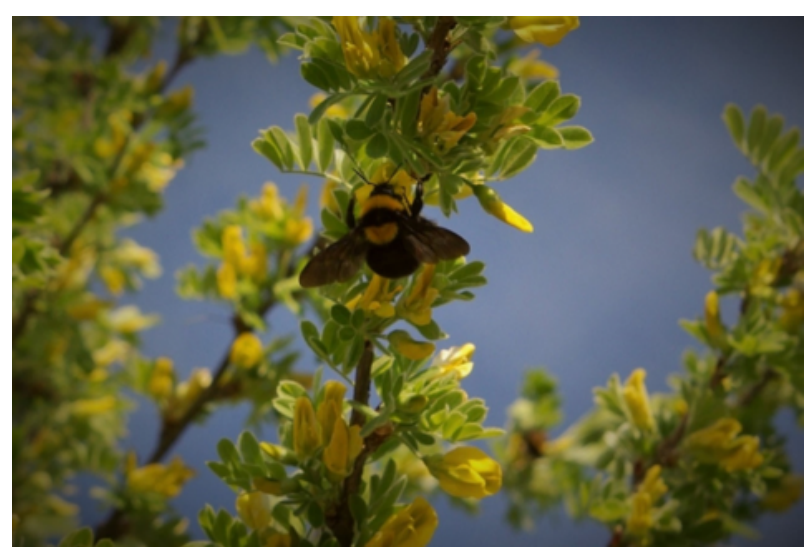

Figure II. Bombus argillaceus sur Caragana arborescens (Fabaceae). (Photo : B. Frin)

\section{Bibliographie}

EDWARdS M, Jenner M, 2018. Field guide of the Bumblebees of Great Britain and Ireland. Countryside and Garden Conservation Series. Ocelli. 108 p.

Chas E., 1994. Atlas de la flore des HautesAlpes. Conservatoire botanique national de GapCharance, Conservatoire des espaces naturels de Provence et des Alpes du sud, Parc National des Ecrins. 816 p.

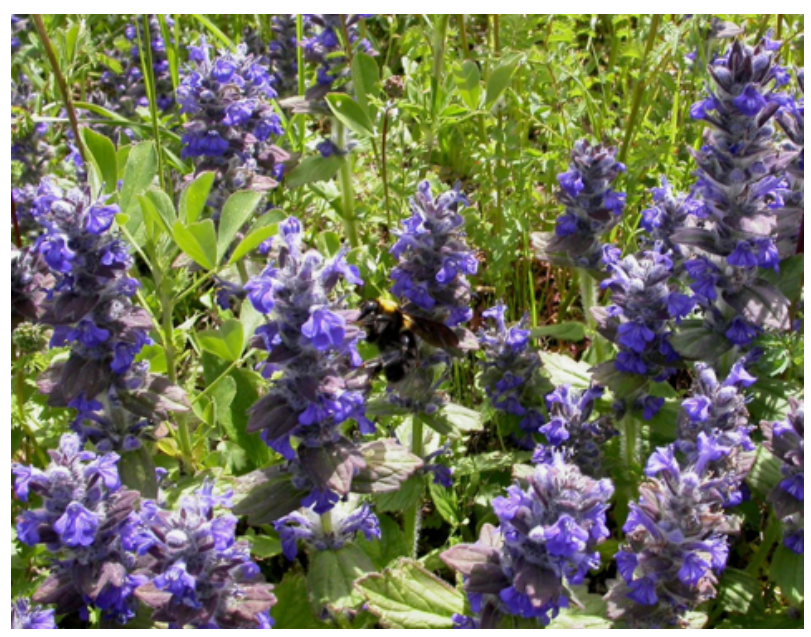

Figure 12 Bombus argillaceus sur Adjuga sp. (Lamiaceae). (Photo : B. Frin) 
Pouvreau A., 2004. Les Insectes Pollinisateurs La Bibliothèque du Naturaliste. Delachaux et Niestlè. 189 p.

RASMONT P, 1988. Monographie écologique et zoogéographique des Bourdons de France et de Belgique. Thèse de doctorat en Sciences Agronomiques, Faculté des Sciences agronomiques de l'Etat, Gembloux, 310 + LXII pp.

MAнÉ G, 2007. Observations de Bombus gerstaeckeri Morawitz (Hymenoptera, Apidae) butinant Delphinium dubium (Rouy et Fouc.) (Ranunculaceae) dans le Massif des Ecrins (Hautes-Alpes, France). Osmia 1 : 16-18.

MAHÉ G, 2008. Bourdons rares du Parc Naturel Régional du Queyras (Hautes-Alpes,France). Osmia $2: 21-25$.

MAHÉ G, 2011. Mémo pour l'atlas des bourdons armoricains. $\quad 11 \mathrm{p} . \quad \mathrm{http}: / /$ www.atlashymenoptera.net/biblio/ Memo2012_Atlas_Bombus_Armor.pdf

RASMONT P \& ISERBYT S, 2010-2013. Atlas of the European Bees: genus Bombus. 3d Edition. STEP Project, Atlas Hymenoptera, Mons, Gembloux. http://www.atlashymenoptera.net/ page.asp?ID=169 